\title{
Lower Approximations by Fuzzy Consequence Operators
}

\author{
J. Elorza ${ }^{1}$ J. Recasens ${ }^{2}$ \\ ${ }^{1}$ Dept. de Física y Matemática Aplicada. Universidad de Navarra, Spain, jelorza@unav.es \\ ${ }^{2}$ Sec. Matemàtiques i Informàtica. ETSAV. U. Politècnica de Catalunya, Spain, j.recasens@upc.edu
}

\begin{abstract}
Three ways to find lower approximations of a given fuzzy operator are given.

A Representation Theorem for fuzzy consequence operators is obtained.
\end{abstract}

Keywords: fuzzy consequence operator, fuzzy closure operator, fuzzy $*$-preorder, $*$-indistinguishability operator, similarity.

\section{Introduction}

Consequence operators are essential in the definition of several logics. In fact, Tarski considered that a logic is defined by giving a universe and a consequence operator on it [19]. In particular, fuzzy consequence operators (FCO) are consequence operators defined on the set of fuzzy subsets of a universe and have been proved essential in the study of mathematical fuzzy logic.

FCOs are not only considered as consequence operators (in order to obtain consequences from a fuzzy predicate) but also have been applied to a wide range of topics. For example, they have been used to generate upper approximations of fuzzy sets in fuzzy rough set theory [15] [3], as closure operators in fuzzy topology [14], to model the fuzzy possibility quantifier in modal logic [6], in the study of fuzzy relational equations [16], in fuzzy mathematical morphology [8], [11] and in fuzzy context theory $[2]$.

There are cases when a given or obtained fuzzy operator $c$ is not a FCO but for technical or theoretical reasons it should be such. In these cases, the operator must be replaced by a FCO. The usual way is replacing $c$ by its closure (see Section 2), which is the smallest FCO greater than or equal to $c$. In this way the best upper approximation of a fuzzy operator by a FCO is obtained.

The question arises whether there can be found the best lower approximation of a fuzzy operator $c$, but the answer is negative because, in general, the supremum of FCOs is not a FCO anymore. We could instead try to find maximal lower approximations of $c$, but this seems a very difficult task. As a realistic alternative, in this paper three methods to find "good" lower approximations of a fuzzy operator by FCOs are provided (Sections 5, 6 and 7).
In Section 2, we recall the basic results needed in the paper. In Section 3, we recall some relationships between Fuzzy Relations and Fuzzy Operators, we generalize the operator induced by a fuzzy relation through Zadeh's compositional rule to a fuzzy operator induced by a fuzzy relation and another fuzzy operator and we introduce the notion of concordance between a fuzzy operator and a fuzzy relation as sufficient condition for inducing fuzzy consequence operators through this generalization. In Section 4, we present the Representation Theorem for FCOs and some consequences of this result will be obtained. In Section 5, using the Representation Theorem for FCOs, we will show lower approximations of a given fuzzy operator $c$ by FCOs. In Section 6 , we present the second lower approximations of a given fuzzy operator $c$ that it is based on the FCO associated with the largest fuzzy preorder $R_{c}^{c}$ for which $c$ can be *-concordant with $R_{c}^{c}$. In Section 7 , the third method is based on the obtention of $*$-transitive openings of fuzzy relations and, as result, a FCO smaller than or equal to $c$ at least in the crisp sets of the universe is obtained. Section 8 with concluding remarks ends this work.

\section{Preliminaries}

A fuzzy operator on a non-empty universe $X$ is a $\operatorname{map} c:[0,1]^{X} \longrightarrow[0,1]^{X}$.

Definition 2.1. A fuzzy operator $c:[0,1]^{X} \longrightarrow$ $[0,1]^{X}$ on $X$ is called a fuzzy consequence operator or fuzzy closure operator (FCO for short) when it satisfies for all $\mu, \nu \in[0,1]^{X}$ :

- Inclusion $\mu \subseteq c(\mu)$

- Monotonicity $\mu \subseteq \nu \Rightarrow c(\mu) \subseteq c(\nu)$

- Idempotence $c(c(\mu))=c(\mu)$,

where inclusion of fuzzy subsets are defined as usual: For $\mu, \nu \in[0,1]^{X}, \mu \subseteq \nu$ if and only if for all $x \in X$ $\mu(x) \leq \nu(x)$.

Fuzzy consequence operators were introduced by Pavelka in 1979 as an extension of Tarski's consequence operators to fuzzy sets [17].

Let us recall the definition of the fuzzy closure of a fuzzy operator. This notion was first defined for general lattices [21] and later translated to the fuzzy context by Pavelka [17]. It can be thought as 
the best upper approximation provided by a fuzzy consequence operator of a given operator.

Definition 2.2. Let $c:[0,1]^{X} \longrightarrow[0,1]^{X}$ be a fuzzy operator on $X$. We define the fuzzy closure $\bar{c}$ of $c$ as the fuzzy operator given by

$$
\bar{c}=\inf _{\substack{\phi \in \Omega \\ c \leq \phi}}\{\phi\} .
$$

where $\Omega$ denotes the set of fuzzy consequence operators on $X$.

The fuzzy closure is uniquely determined and it is a fuzzy consequence operator since the infimum of fuzzy consequence operators so is.

Definition 2.3. Let $*$ be a t-norm. A fuzzy relation $R: X \times X \longrightarrow[0,1]$ on a universe $X$ is called a fuzzy *-preorder if it satisfies $\forall x, y, z \in X$ :

- Reflexivity: $R(x, x)=1$

- *-Transitivity: $R(x, y) * R(y, z) \leq R(x, z)$.

A fuzzy *-preorder is called a fuzzy *-indistinguishability operator if it also satisfies

- Symmetry: $R(x, y)=R(y, x) \quad \forall x, y \in X$

We will consider the sup-* composition of fuzzy relations.

Definition 2.4. Let $R, S$ be fuzzy relations on a set $X$ and $*$ a t-norm. The sup-* composition $R \circ S$ of $R$ and $S$ is the fuzzy relation defined for all $x, y \in X$ by

$$
R \circ S(x, y)=\sup _{z \in X}\{R(x, z) * S(z, y)\}
$$

Due to associativity of t-norms, for continuous tnorms we can define $R^{n}$ for every fuzzy relation $R$ on $X$ and $n \in \mathbb{N}$.

Definition 2.5. Let $*$ be a t-norm and $R$ a fuzzy relation on a set $X$. The *-transitive closure $\bar{R}$ of $R$ is the fuzzy relation $\bar{R}$ of $X$ satisfying

- $R<\bar{R}$

- If $S$ is another $*$-transitive fuzzy relation on $X$ satisfying $R \leq S$, then $\bar{R} \leq S$.

Proposition 2.6. [13] For a continuous t-norm *, the transitive closure $\bar{R}$ of a fuzzy relation $R$ on $X$ is $\bar{R}=\sup _{n \in \mathbb{N}} R^{n}$.

Proposition 2.7. [13] If $R$ is a reflexive fuzzy relation on $X$, then its $*$-transitive closure $\bar{R}$ is a fuzzy *-preorder on $X$.

Definition 2.8. For a given -norm $*$ and a given fuzzy relation $R$ on $X$, a fuzzy subset $\mu$ of $X$ is called *-compatible with $R$ if and only if $\mu(x) * R(x, y) \leq$ $\mu(y)$ for all $x, y \in X$.

This notion gets special interest when $R$ is a fuzzy *-preorder [7]. When $R$ is an indistinguishability operator, these sets are called extensional sets and they have been largely studied [18].

\section{Connections between Fuzzy Relations and Fuzzy Operators}

Concepts of fuzzy relations and fuzzy operators are closely related. Every fuzzy relation $R$ induces a fuzzy operator $c_{R}$ through the well-known Zadeh's rule of inference [22].

Definition 3.1. Let $R$ be a fuzzy relation on $X$. The fuzzy operator $c_{R}$ induced by $R$ through Zadeh's compositional rule is defined by

$$
c_{R}(\mu)(x)=\sup _{y \in X}\{\mu(y) * R(y, x)\}
$$

Notice that from a logical point of view, $c_{R}$ can be understood as the operator that sends every fuzzy set $\mu$ to the fuzzy set containing all the elements which are related to some element $y$ in $\mu$ by means of the relation $R$.

Proposition 3.2. [10] Let $\sigma$ be the function that sends every fuzzy relation $R$ to the operator $c_{R}$ induced by means of equation (1). Then, $\sigma$ is injective.

In other words, injectivity of $\sigma$ states that for any two fuzzy relations $R$ and $S$, we have $c_{R}=c_{S}$ if and only if $R=S$. The relationship between fuzzy *preorders and fuzzy consequence operators is well established [9] [7].

Proposition 3.3. [9] Let $R$ be a fuzzy relation. Then $c_{R}$ is a fuzzy consequence operator if and only if $R$ is a fuzzy *-preorder.

It is worth recalling that not all FCO can be obtained from fuzzy *-preorders by means of Zadeh's compositional rule.

When the starting relation is a fuzzy indistinguishability operator, the induced operator is not only a FCO but satisfies the following properties [5] [18].

Proposition 3.4. [5] Let $E$ be a fuzzy *-indistinguishability operator and let $c_{E}$ be the fuzzy operator induced through Zadeh's compositional rule. Then,

- $c_{E}$ is a fuzzy consequence operator.

- $c_{E}\left(\bigvee_{i \in I} \mu_{i}\right)=\bigvee_{i \in I} c_{E}\left(\mu_{i}\right)$ for any index set I and all $\mu_{i} \in[0,1]^{X}$.

- $c_{E}(\{x\})(y)=c_{E}(\{y\})(x)$ for all $x, y \in X$ where $\{x\}$ denotes the singleton of $x$.

- $c_{E}(\alpha * \mu)=\alpha * c_{E}(\mu)$ for any constant $\alpha \in[0,1]$ and $\mu \in[0,1]^{X}$.

Proposition 3.5. [5] There is a bijection between the set of $*$-indistinguishability operators and the set of fuzzy operators satisfying the conditions of Proposition 3.4.

We generalize the operator induced by a fuzzy relation through Zadeh's compositional rule to a fuzzy operator induced by a fuzzy relation and another fuzzy operator. 
Definition 3.6. Let $g$ be a fuzzy operator and let $R$ be a fuzzy relation on $X$. We define the operator $c_{R}^{g}$ induced by $g$ and $R$ as

$$
c_{R}^{g}(\mu)(x)=\sup _{y \in X}\{g(\mu)(y) * R(y, x)\}
$$

$R$ and $g$ are called the generators of $c_{R}^{g}$.

The operator $g$ used as generator performs a selection in order to apply Zadeh's usual operator only to the fuzzy subsets of its image. Notice that taking $g=i d$, where $i d$ denotes the identity operator on $[0,1]^{X}$, we obtain $c_{R}^{i d}=c_{R}$.

Proposition 3.7. For every fuzzy operator $g$ on $X$, the mapping $\sigma_{g}$ that sends every fuzzy relation $R$ to the operator $c_{R}^{g}$ induced by $R$ and $g$ by means of equation (2) is increasing. That is, if $R \leq S$ then $c_{R}^{g} \leq c_{S}^{g}$.

Proof. It directly follows from the monotonicity of *.

Corollary 3.8. The mapping $\sigma$ that sends every fuzzy relation $R$ to the operator $c_{R}$ induced by Zadeh's compositional rule (equation (1)) is increasing.

Our interest lies in the obtention of fuzzy consequence operators. For this, we need certain individual properties of the generators and also some conditions involving both generators, operators and relations. More precisely, let us define the concordance between a fuzzy operator and a fuzzy relation.

Definition 3.9. [4] Let $g$ be a fuzzy operator and $R$ a fuzzy relation. We will say that $g$ is *-concordant with $R$ if and only if all the subsets from the image of $g$ are *-compatible with $R$. That is,

$$
g(\mu)(x) * R(x, y) \leq g(\mu)(y)
$$

for all $x, y \in X$ and all $\mu \in[0,1]^{X}$.

Theorem 3.10. Let $R$ be a reflexive fuzzy relation and let $g$ be a FCO on $X$. Suppose that $g$ is *concordant with $R$. Then, the operator $c_{R}^{g}$ induced by $g$ and $R$ is also a FCO.

Proof. Let us start proving the inclusion and monotonicity properties. From the reflexivity of $R$, it follows that

$$
\begin{aligned}
c_{R}^{g}(\mu)(x) & =\sup _{y \in X}\{g(\mu)(y) * R(y, x)\} \\
& \geq g(\mu)(x) * R(x, x)=g(\mu)(x) .
\end{aligned}
$$

Since $g$ is a FCO and therefore inclusive, we get

$$
c_{R}^{g}(\mu)(x) \geq g(\mu)(x) \geq \mu(x)
$$

Let $\mu_{1}, \mu_{2}$ be fuzzy subsets of $X$ such that $\mu_{1} \subseteq$ $\mu_{2}$. From the monotonicity of $g$ it follows that $g\left(\mu_{1}\right)(x) \leq g\left(\mu_{2}\right)(x)$ for all $x \in X$. Therefore,

$$
\begin{aligned}
c_{R}^{g}\left(\mu_{1}\right)(x) & =\sup _{y \in X}\left\{g\left(\mu_{1}\right)(y) * R(y, x)\right\} \\
& \leq \sup _{y \in X}\left\{g\left(\mu_{2}\right)(y) * R(y, x)\right\}=c_{R}^{g}\left(\mu_{2}\right)(x) .
\end{aligned}
$$

It only remains to prove the idempotence. a) $c_{R}^{g} \circ c_{R}^{g} \leq c_{R}^{g}$ :

Since $g(\mu)$ belongs to $\operatorname{Im}(g)$, it is $*$-compatible with $R$. That is,

$$
g(\mu)(y) * R(y, x) \leq g(\mu)(x)
$$

for all $y, x \in X$. Hence,

$$
\sup _{y \in X}\{g(\mu)(y) * R(y, x)\} \leq g(\mu)(x)
$$

for all $x \in X$. Using this fact, the monotonicity and idempotence of $g$ and the monotonicity of * we get

$$
\begin{aligned}
c_{R}^{g}\left(c_{R}^{g}(\mu)\right)(x) & \\
= & \sup _{y \in X}\left\{g\left(C_{R}^{g}(\mu)\right)(y) * R(y, x)\right\} \\
= & \sup _{y \in X}\left\{g\left(\sup _{z \in X}\{g(\mu)(z) * R(z, y)\}\right) * R(y, x)\right\} \\
\leq & \sup _{y \in X}\{g(g(\mu)(y)) * R(y, x)\} \\
= & \sup _{y \in X}\{g(\mu)(y) * R(y, x)\}=c_{R}^{g}(\mu)(x)
\end{aligned}
$$

b) $c_{R}^{g} \circ c_{R}^{g} \geq c_{R}^{g}$ :

This inclusion follows immediately from the inclusion property.

\section{Representation Theorem for Fuzzy Consequence Operators}

The fact that every fuzzy subset $\mu$ of a universe $X$ generates a FCO in a natural way will allow us to establish a representation theorem for such operators.

Definition 4.1. Given a fuzzy subset $\mu$ of $X$, we define the fuzzy operator $c_{\mu}:[0,1]^{X} \rightarrow[0,1]^{X}$ on $X$ by

$$
c_{\mu}(\nu)= \begin{cases}\mu & \text { if } \nu \leq \mu \\ 1 & \text { otherwise }\end{cases}
$$

where $1(x)=1$ for all $x \in X$.

Proposition 4.2. $c_{\mu}$ is a fuzzy consequence operator on $X$.

Proof. Straightforward.

Definition 4.3. Given a family $M=\left(\mu_{i}\right)_{i \in I}$ of fuzzy subsets of $X, c_{M}$ is the fuzzy operator on $X$ defined by

$$
c_{M}=\inf _{i \in I} c_{\mu_{i}}
$$

Proposition 4.4. $c_{M}$ is a fuzzy consequence operator on $X$.

Proof. The infimum of FCOs is a FCO. 
Theorem 4.5. Representation Theorem of Fuzzy Consequence Operators. Let $c$ : $[0,1]^{X} \rightarrow[0,1]^{X}$ be a fuzzy operator on $X$. Then $c$ is a fuzzy consequence operator if and only if there exists a family $M$ of fuzzy subsets of $X$ such that $c=c_{M}$

Proof.

$\Rightarrow$ Let $M$ be the family of all fuzzy subsets that are in the image of $c$, i.e.: $M=\left\{c(\mu) \mid \mu \in[0,1]^{X}\right\}$. We will prove that $c_{M}=c$. Indeed,

$c_{M} \leq c$ :

$$
c_{M}(\mu) \leq c_{c(\mu)}(\mu)=c(\mu) .
$$

$c \leq c_{M}$ : For a fixed fuzzy subset $\mu$,

if $\mu \not \leq c(\nu)$, then $c_{c(\nu)}(\mu)=\mathbb{1}$ and

if $\mu \leq c(\nu)$, then $c(\mu) \leq c(c(\nu))=c(\nu)$.

Hence in any case,

$$
c(\mu) \leq c_{c(\nu)}(\mu)
$$

and

$$
c(\mu) \leq \inf _{\rho \in M} c_{\rho}(\mu)=c_{M}(\mu) .
$$

$\Leftarrow)$ Proposition 4.4 .

Theorem 4.5 suggests the following definition.

Definition 4.6. The infimum of the cardinalities of families $M$ generating $c$ is called its dimension. Such a family is called a basis of $c$.

Example 4.7. Given a fuzzy subset $\mu$ of $X, c_{\mu}$ is a one-dimensional fuzzy consequence operator.

Note that in general $c_{\mu} \wedge c_{\nu} \neq c_{\mu \wedge \nu}$.

Proposition 4.8. $c_{\mu} \wedge c_{\nu}$ is one-dimensional if and only if $\mu \leq \nu$ or $\nu \leq \mu$.

Proof.

$\Rightarrow)$

If $\mu \not \leq \nu$ and $\nu \not \leq \mu$, then there exist $x_{0}, x_{1} \in X$ such that $\mu\left(x_{0}\right)<\nu\left(x_{0}\right)$ and $\nu\left(x_{1}\right)<\mu\left(x_{1}\right)$.

Consider the fuzzy subsets $\tau_{i}, i=0,1$ defined by

$$
\tau_{i}(z)= \begin{cases}0 & \text { if } z \neq x_{i} \\ \frac{\mu(z)+\nu(z)}{2} & \text { if } z=x_{i}\end{cases}
$$

$c_{\mu}\left(\tau_{0}\right)=\mathbb{1}$ and $c_{\nu}\left(\tau_{0}\right)=\nu$ and $\left(c_{\mu} \wedge c_{\nu}\right)\left(\tau_{0}\right)=\nu$. $c_{\mu}\left(\tau_{1}\right)=\mu$ and $c_{\nu}\left(\tau_{1}\right)=1$ and $\left(c_{\mu} \wedge c_{\nu}\right)\left(\tau_{1}\right)=\mu$. $\Leftarrow)$

Hence $c_{\mu} \wedge c_{\nu}$ is not one-dimensional.

If $\mu \leq \nu$, then $c_{\mu} \leq c_{\nu}$ and $c_{\mu} \wedge c_{\nu}=c_{\mu}$.

Similarly, if $\nu \leq \mu$, then $c_{\mu} \wedge c_{\nu}=c_{\nu}$.

Definition 4.9. For a fuzzy consequence operator $c, H_{c}=\left\{c(\mu) \mid \mu \in[0,1]^{X}\right\}$.
Remark 4.10. $H_{c}$ is the set of fixed points of the operator $c$.

Proposition 4.11. $H_{c}$ is a basis of $c$.

Corollary 4.12. If $H_{c}$ is finite, then the basis of a fuzzy consequence operator is unique.

Remark 4.13. If $H_{c}$ has a non-finite cardinality, the last claim needs not to be true. The following very simple example illustrates this fact.

Example 4.14. Consider the family $\left(\mu_{i}\right)_{i \in\left[\frac{1}{2}, 1\right]}$ of fuzzy subsets of $X=\{a\}$ defined for all $i \in\left[\frac{1}{2}, 1\right]$ by

$$
\mu_{i}(a)=i
$$

This family generates a family $\left(c_{i}\right)_{i \in\left[\frac{1}{2}, 1\right]}$ of FCOs on $X$ and is a basis of the fuzzy consequence operator $c=\inf _{i \in\left(\frac{1}{2}, 1\right]} c_{i}$.

But the family $\left(\mu_{i}\right)_{i \in\left(\frac{1}{2}, 1\right]}$ (excluding the fuzzy subset $\left.\mu_{\frac{1}{2}}\right)$ of $X$ also generates $c$ and is therefore another basis of $c$.

\section{Lower Approximation with the Representation Theorem}

Given a fuzzy inclusive and monotone operator $c$ on $X$ and $\mu$ a fuzzy subset of $X$, we consider the operator $c_{c(\mu)}$ :

$$
c_{c(\mu)}(\nu)= \begin{cases}c(\mu) & \text { if } \nu \leq c(\mu) \\ \mathbb{1} & \text { if } \nu \leq c(\mu) .\end{cases}
$$

Proposition 5.1. Given a fuzzy inclusive and monotone operator $c$ and $\mu$ a fuzzy subset of $X$, the operator

$$
c^{\prime}=\inf _{\mu \in[0,1]^{X}} c_{c(\mu)}
$$

satisfies

1. $c^{\prime}$ is a FCO.

2. $c^{\prime} \leq c$.

Proof.

1. Each $c_{c(\mu)}$ is a fuzzy consequence operator and the infimum of FCOs is also another FCO.

2. Given a fuzzy subset $\nu$ of $X$,

$$
c^{\prime}(\nu) \leq c_{c(\nu)}(\nu)=c(\nu)
$$

In this way a lower approximation of $c$ by the FCO $c^{\prime}$ is obtained.

Corollary 5.2. $c=c^{\prime}$ if and only of $c$ is a FCO. 


\section{Lower Approximation associated with a Fuzzy *-preorder}

Proposition 6.1. Let $*$ be a t-norm and $c$ : $[0,1]^{X} \rightarrow[0,1]^{X}$ a fuzzy operator on $X$. The fuzzy relation $R_{c}^{c}$ on $X$ defined for all $x, y \in X$ by

$$
R_{c}^{c}(x, y)=\inf _{\mu \in[0,1]^{X}}\left\{c(\mu)(x) \rightarrow_{*} c(\mu)(y)\right\}
$$

is a fuzzy *-preorder, where $\rightarrow_{*}$ is the residuum of $*$, defined for all $x, y \in[0,1]$ by $x \rightarrow_{*} y=\sup \{\alpha \in$ $[0,1] \mid x * \alpha \leq y\}$.

Proof. It is a consequence of the Representation Theorem for fuzzy $*$-preorders [20].

Consider the FCO $c_{R_{c}^{c}}$ associated with $R_{c}^{c}$ defined by

$$
c_{R_{c}^{c}}(\mu)(x)=\sup _{y \in X}\left\{\mu(y) * R_{c}^{c}(y, x)\right\} .
$$

This FCO is a lower approximation of $c$ as will be stated and proved in the next Proposition 6.3.

Lemma 6.2. [1] Given a left-continuous t-norm * and $a, b \in[0,1], a *\left(a \rightarrow_{*} b\right) \leq b$, where $a \rightarrow_{*} b$ is the residuum of the $t$-norm $*$.

Proposition 6.3. For a fuzzy inclusive operator $c$ on $X, c_{R_{c}^{c}} \leq c$.

Proof.

$$
\begin{aligned}
c_{R_{c}^{c}} & (\mu)(x) \\
= & \sup _{y \in X}\left\{\mu(y) * R_{c}^{c}(y, x)\right\} \\
= & \sup _{y \in X}\left\{\mu(y) * \inf _{\nu \in[0,1]^{X}}\left\{c(\nu)(y) \rightarrow_{*} c(\nu)(x)\right\}\right\} \\
\leq & \sup _{y \in X}\left\{\mu(y) *\left(c(\mu)(y) \rightarrow_{*} c(\mu)(x)\right\}\right. \\
\leq & \sup _{y \in X}\left\{c(\mu)(y) *\left(c(\mu)(y) \rightarrow_{*} c(\mu)(x)\right\}\right. \\
\leq & c(\mu)(x)
\end{aligned}
$$

where the last inequality follows from Lemma 6.2.

Example 6.4. Consider the fuzzy operator $c$ : $[0,1]^{[0,1]} \rightarrow[0,1]^{[0,1]}$ on $X=[0,1]$ defined for all fuzzy subset of $[0,1]$ and for all $x \in[0,1]$ by

$$
c(\mu)(x)=\min \left\{1, \mu(x)+\frac{1}{2}\right\} .
$$

The fuzzy operator $c$ is inclusive and monotone but not idempotent. In this simple example, $R_{c}^{c}$ is independent of the chosen t-norm.

$$
\begin{aligned}
R_{c}^{c}(x, y) & =\inf _{\mu \in[0,1]^{[0,1]}}\left\{c(\mu)(x) \rightarrow_{*} c(\mu)(y)\right\} \\
& = \begin{cases}\frac{1}{2} & \text { if } x \neq y \\
1 & \text { if } x=y .\end{cases}
\end{aligned}
$$

$$
\begin{aligned}
c_{R_{c}^{c}}(\mu)(x) & =\max \left\{\sup _{y \neq x}\left\{\mu(y) * \frac{1}{2}\right\}, \mu(x)\right\} \\
& = \begin{cases}\frac{1}{2} & \text { if } \mu(x) \leq \frac{1}{2} \\
\mu(x) & \text { if } \mu(x)>\frac{1}{2} .\end{cases}
\end{aligned}
$$

In Figure 1 the fuzzy subsets $\mu(x)=0.5 x+0.2$, $c(\mu)$ and $c_{R_{c}^{c}}(\mu)$ of $[0,1]$ are plotted in blue, black and red respectively.

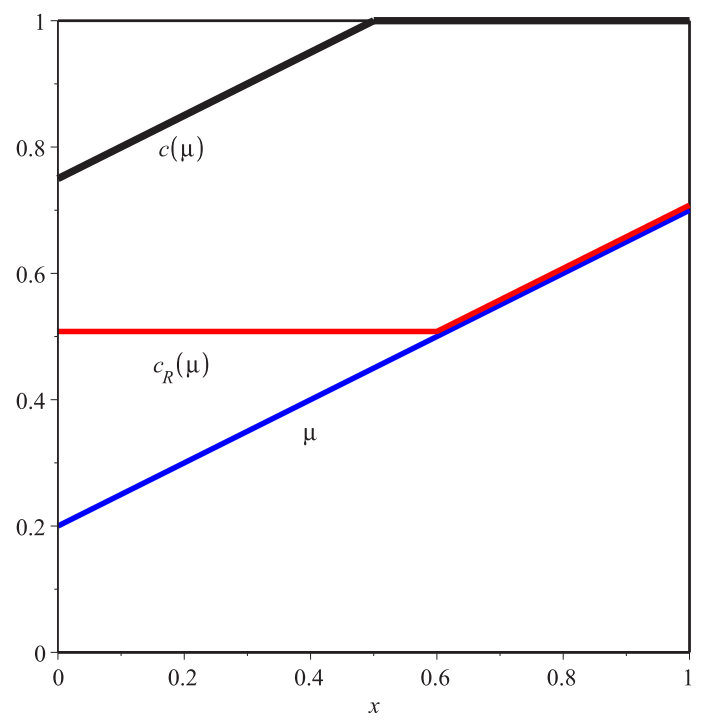

Figure 1: $\mu(x)=0.5 x+0.2, c(\mu)$ and $c_{R_{c}^{c}}(\mu)$.

\section{Lower Approximation with $*$-transitive Openings}

In this section a way to find a FCO $\underline{c}$ from a given inclusive fuzzy operator $c$ on $X$ that is below $c$ for the crisp subsets of $X$ (i.e.: $\underline{c}(A) \leq c(A)$ for all $A \subseteq X)$ will be provided. It is based on the *transitive openings of a fuzzy relation (Definition 7.1).

The transitive closure of a reflexive fuzzy relation $R$ gives a fuzzy *-preorder greater than or equal to $R$. In this case it is possible to obtain the best upper approximation since the infimum of fuzzy $*$-preorders is also a fuzzy $*$-preorder. If we want a lower approximation, then the situation is more complicated since the supremum of fuzzy *preorders is not a fuzzy $*$-preorder in general. What we can find are fuzzy *-preorders which are maximal among the ones that are smaller than or equal to a given reflexive fuzzy relation. These relations are called $*$-transitive openings and they are not unique in general. In fact, there can be an infinite quantity of them, even in sets of finite cardinality.

In [12], an algorithm to find maximal transitive openings of a given fuzzy relation is given that will be used in this section. 
Definition 7.1. Let $R$ be a reflexive fuzzy relation on a set $X$ and $*$ a t-norm. A fuzzy $*$-preorder $\underline{R}$ on $X$ is $a *$-transitive opening of $R$ if and only if

- $\underline{R} \leq R$

- If $P$ is another fuzzy $*$-preorder on $X$ satisfying $P \leq R$, then $P \leq \underline{R}$.

Let $c:[0,1]^{X} \rightarrow[0,1]^{X}$ be an inclusive fuzzy operator on $X$. Then the fuzzy relation $R_{c}$ on $X$ defined by $R_{c}(x, y)=c(\{x\})(y)$ for all $x, y \in X$ is reflexive. For a continuous Archimedean t-norm * we can calculate a $*$-transitive opening $\underline{R}$ of $R_{c}$ if $X$ is a finite set [12]. From $\underline{R}$ we can calculate its associated FCO $c_{\underline{R}}$ which satisfies $c_{\underline{R}}(A) \leq c(A)$ for all crisp subsets $\bar{A}$ of $X$.

Example 7.2. Consider an inclusive and monotone fuzzy operator $c$ on $X=\left\{x_{1}, x_{2}, x_{3}, x_{4}\right\}$ generating the fuzzy relation $R$ with matrix

$$
R=\left(\begin{array}{cccc}
1 & 0.75 & 0.70 & 0.62 \\
0.76 & 1 & 0.90 & 1 \\
0.70 & 0.86 & 1 & 1 \\
0.64 & 0.65 & 0.94 & 1
\end{array}\right)
$$

(This means that the entry $r_{i j}, i \leq i, j \leq 4$, of $R$ is $\left.c\left(\left\{x_{i}\right\}\right)\left(x_{j}\right)\right)$. In [12] the following *-transitive opening $\underline{R}$ of $R$ for the t-norm $\min$ is obtained.

$$
\underline{R}=\left(\begin{array}{cccc}
1 & 0.62 & 0.62 & 0.62 \\
0.76 & 1 & 0.90 & 1 \\
0.64 & 0.64 & 1 & 1 \\
0.64 & 0.62 & 0.90 & 1
\end{array}\right)
$$

Its associated $\mathrm{FCO} c_{\underline{R}}$ on $X$ is smaller than or equal to $c$ on the crisp sets of $X$.

\section{Concluding Remarks}

In this work the problem of approximating a fuzzy operator $c$ by a FCO smaller than or equal to $c$ is tackled. Three different ways to do so have been provided. The first one is based on a stated Representation Theorem for FCOs, the second one on fuzzy $*$-preorders and the last one on $*$-transitive openings of fuzzy relations. Also a Representation Theorem for FCOs stating that every FCO can be generated by a family of fuzzy subsets is proved. The problem of finding maximal lower approximations of a fuzzy operator, though very interesting, seems very difficult. The authors plan to continue its study in forthcoming works.

\section{References}

[1] Bělohlávek, R., 2002. Fuzzy Relational Systems: Foundations and Principles. IFSR international series on systems science and engineering. Kluwer Academic/Plenum Publishers.

[2] Bělohlávek, R., 2004. Concept lattices and order in fuzzy logic. Annals of Pure and Applied Logic 128 (13), 277-298.
[3] Boixader, D., Jacas, J., Recasens, J., 2000. Upper and lower approximations of fuzzy sets. International Journal of General Systems 29 (4), 555-568.

[4] Carmona, N., Elorza, J., Recasens, J. Bragard, J. Permutable Fuzzy Consequence and Interior Operators and their Connection with Fuzzy Relations. Information Sciences (2015), http://dx.doi.org/10.1016/j.ins.2015.03.017

[5] Castro, J.L., Klawonn, F., 1996. Similarity in Fuzzy Reasoning. Mathware \& Soft Computing 2, 197-228.

[6] Bou, F., Godo, L., Esteva, F., Rodriguez, R. O., 2011. On the minimum many-valued modal logic over a finite residuated lattice. Journal of Logic and Computation 5 (21), 739-790.

[7] Castro, J., Delgado, M., Trillas, E., 1994. Inducing implication relations. International Journal of Approximate Reasoning 10 (3), 235250.

[8] De Baets, B., Kerre, E., Gupta, M., 1995. The fundamentals of fuzzy mathematical morphology. Part 1: Basic concepts. International Journal of General Systems 23 (2), 155-171.

[9] Elorza, J., Burillo, P., 1999. On the relation between fuzzy preorders and fuzzy consequence operators. International Journal of Uncertainty, Fuzziness and Knowledge-Based Systems 7 (3), 219-234.

[10] Elorza, J., Burillo, P., 2003. Connecting fuzzy preorders, fuzzy consequence operators and fuzzy closure and co-closure systems. Fuzzy Sets and Systems 139 (3), 601-613.

[11] Elorza, J., Fuentes-González, R., Bragard, J. Burillo, P., 2013 On the relation between fuzzy closing morphological operators, fuzzy consequence operators induced by fuzzy preorders and fuzzy closure and co-closure systems. Fuzzy Sets and Systems, Vol. 218, 73-89.

[12] Fodor, J.C., Roubens, M., 1995. Structure of transitive valued binary relations. Math. Soc. Sci. 30, 71-94.

[13] Klir, G.J., Yuan, B., Fuzzy sets and fuzzy logic: Theory and applications. Prentice Hall (1995)

[14] Lowen, R., 1976. Fuzzy topological spaces and fuzzy compactness. Journal of Mathematical Analysis and Applications 56 (3), 621-633.

[15] Morsi, N. N., Yakout, M. M., 1998. Axiomatics for fuzzy rough sets. Fuzzy Sets and Systems 100 (1-3), 327-342.

[16] Di Nola, A., Sessa, S., Pedrycz, W., Sanchez, E., 1989. Fuzzy Relation Equations and Their Applications to Knowledge Engineering. Theory and decision library: System theory, knowledge engineering, and problem solving. Kluwer Academic Publishers, Springer.

[17] Pavelka, J., 1979. On fuzzy logic I: Manyvalued rules of inference. Mathematical Logic Quarterly 25 (3-6), 45-52.

[18] Recasens, J., 2011. Indistinguishability Oper- 
ators: Modelling Fuzzy Equalities and Fuzzy Equivalence Relations. Vol. 260 of Studies in Fuzziness and Soft Computing. Springer.

[19] Tarski, A. Logic, semantics, metamathematics. Clarendon Press, Oxford, 1956.

[20] Valverde, L., 1985. On the Structure of Findistinguishability Operators. Fuzzy Sets and Systems 17, 313-328.

[21] Ward, M., 1940. The closure operators of a lattice. Annals of Mathematics 43 (2), 191-196.

[22] Zadeh, L. A., 1975. The concept of a linguistic variable and its application to approximate reasoning - III. Information Sciences 9 (1), 4380. 